\title{
Dependence of the perpendicular anisotropy in Co/Au multilayers on the number of repetitions
}

\author{
G. Gubbiottia) and G. Carlotti \\ INFM, Unità di Perugia and Dipartimento di Fisica, Via A. Pascoli, 06123 Perugia, Italy \\ F. Albertini \\ IMEM-CNR, Parco Area delle Scienze 37/A, 43010 Fontanini, Parma, Italy \\ F. Casoli, E. Bontempi, and L. E. Depero \\ INSTM \& Laboratorio di Strutturistica Chimica, Dipartimento di Ingegneria Meccanica, \\ Università di Brescia, Via Branze, 38, 25123 Brescia, Italy \\ P. Mengucci and A. Di Cristoforo \\ INFM-Unità di Ancona, Dipartimento di Fisica e Ingegneria dei Materiali e del Territorio, \\ Facoltà di Ingegneria Università degli Studi di Ancona, Via Brecce Bianche, I-60131, Ancona, Italy \\ H. Koo and R. D. Gomez \\ Department of Electrical and Computer Engineering, University of Maryland, College Park, Maryland 20742
}

(Presented on 13 November 2002)

The correlations between structure and magnetism in $[\mathrm{Co}(0.9 \mathrm{~nm}) / \mathrm{Au}(5 \mathrm{~nm})] \times N$ multilayer films
with different number of repetitions $N=10,30$, and 50 , have been studied by the combined use of
complementary structural and magnetic techniques, such as X-ray reflectivity, x-ray diffraction, and
transmission electron microscopy, alternating gradient force magnetometry, magnetic force
microscopy and Brillouin light scattering. On increasing the value of $N$, an overall improvement of
the multilayer quality is observed which corresponds to a change in the micromagnetic structure and
to an enhancement of the perpendicular anisotropy. These effects have been attributed to a reduction
of the magnetostatic energy associated with the formation of perpendicular magnetic domains in
multilayers with increasing number of layers repetitions. C 2003 American Institute of Physics. [DOI: 10.1063/1.1557314]

Perpendicular magnetic anisotropy (PMA) is a peculiar property of magnetic multilayers, whose interest is mainly due to its possible exploitation in the field of magnetic and magneto-optic recording. ${ }^{1}$ In previous articles, we have shown that in $\mathrm{Co} / \mathrm{Au}$ multilayers PMA is strongly influenced not only by the elemental thickness ${ }^{2}$ but also by the annealing treatments which affect the interface quality. ${ }^{3}$ Another interesting effect has been observed in $\mathrm{Co} / \mathrm{Au}$ multilayers by Hamada et $\mathrm{al}^{4}{ }^{4}$ who performed a detailed investigation of the magnetic properties as a function of the number of layer repetitions. On increasing the number of bilayer repetitions $N$, a smooth spin reorientation transition from in-plane to out-of-plane has been observed. In this work, results concerning the evolution of structure, magnetic microstructure, and magnetic anisotropy in $\mathrm{Co} / \mathrm{Au}$ multilayers as a function of the number of repetitions are reported. This study has been carried out by the combined use of complementary techniques, such as diffraction and reflectivity of $\mathrm{x}$ rays, transmission electron microscopy (TEM), alternating gradient force magnetometry (AGFM), magnetic force microscopy, and Brillouin light scattering (BLS) from spin waves.

$\mathrm{Co} / \mathrm{Au}$ multilayer films were sputtered by means of a rf system with a base pressure of $2 \times 10^{-8} \mathrm{mbar}$ and a working Ar pressure of $2.9 \times 10^{-2}$ mbar. $^{2,3}$ The multilayers were deposited with the same thickness of the elemental layers $\left(t_{\mathrm{Co}}\right.$

${ }^{a)}$ Author to whom correspondence should be addressed; electronic mail: gubbiotti@fisica.unipg.it $\left.=0.9 \mathrm{~nm}, t_{\mathrm{Au}}=5 \mathrm{~nm}\right)$ and different numbers of Co/Au bilayers $(N=10,30$, and 50). The thickness of the $\mathrm{Co}$ and $\mathrm{Au}$ layers was chosen in order to have perpendicular anisotropy ${ }^{2}$ and negligible interlayer exchange coupling, ${ }^{5}$ respectively. The films were covered with an Au capping layer to avoid the Co oxidation.

The structural and morphological characterization of the samples was accomplished by transmission electron microscopy, x-ray reflectivity (XRR) and x-ray diffraction (XRD).

A complete analysis of the magnetic properties was realized by employing three different magnetic techniques. The magnetization loops were measured at room temperature by an alternating gradient-force magnetometer (AGFM) with the applied magnetic field either parallel or perpendicular to the sample surface. This kind of characterization was supported by the observation of the perpendicular magnetic domains by magnetic force microscopy (MFM). Brillouin light scattering (BLS) has been employed to measure the field dependence of spin waves in order to determine the effective anisotropy constant. BLS experiments were performed in the backscattering configuration at the GHOST laboratory, ${ }^{6}$ University of Perugia, using a Sandercock-type $(3+3)$-pass interferometer. A dc magnetic field was applied in the sample plane perpendicularly to the incidence plane of light.

In Fig. 1(a), the TEM micrograph for the $N=50$ multilayer shows the whole film where the different layers are clearly visible. The top surface of the multilayer is wavy and this effect is due to the formation of columnar grains 


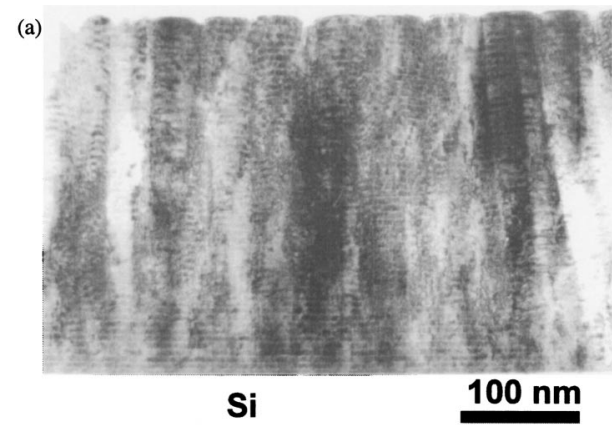

(b)

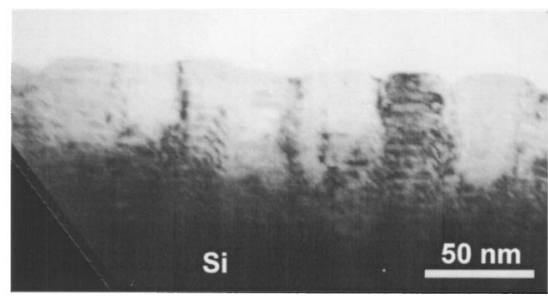

FIG. 1. Cross section TEM micrograph for the Co/Au multilayer with (a) $N=50$ and (b) $N=30$ bilayer repetitions, respectively.

through the entire thickness of the deposited film. Although this columnar grain structure is almost well defined, the different layers are still clearly visible indicating that intermixing effects between $\mathrm{Au}$ and $\mathrm{Co}$, if present, are limited to the interface regions. In other words, the layered structure of the film is maintained in despite of the incipient formation of the columnar grains. These grains have lateral dimensions ranging from 40 to $50 \mathrm{~nm}$, as evidenced in the micrograph. The formation of these grains is also responsible of the wavy interfaces between the alternated $\mathrm{Au}$ and Co layers. The mean thickness of the Co layer is about $1 \mathrm{~nm}$ whereas that of the Au layer is about $4.5 \mathrm{~nm}$. For the sample with $N=30$, see Fig. 1(b), the analysis of the TEM micrographs indicates that the grains start to appear at the interface with the Si substrate and in correspondence of the larger columnar grains the alternated layers appear much more bent and the layers' thickness is not constant. Furthermore, inside the same columnar grain the interfaces between $\mathrm{Au}$ and $\mathrm{Co}$ are rough. Since the grain's dimensions are almost the same for all the samples, we can conclude that the local strain, induced on the whole multilayer by the grains formation, is larger for the specimens with a lower value of $N$.

The experimental and simulated XRR spectra of the three multilayers are reported in Fig. 2. A qualitative examination of the spectra confirms the presence of disorder in the layered structure, as already evidenced from the TEM measurements. In fact, the Bragg peaks are broad and the effect is more evident in the thinnest multilayer $(N=10)$. In addition, the peaks' broadening increases at high angles since the high order Bragg peaks are more sensitive to variations in the multilayer periodicity. The XRR patterns were simulated by the program REFSIM ${ }^{7}$ that allows to deduce layer thickness, surface, and interface roughness of the multilayers. The simulations present some discrepancies from the experimental data, probably due to the waviness of the multilayer struc-

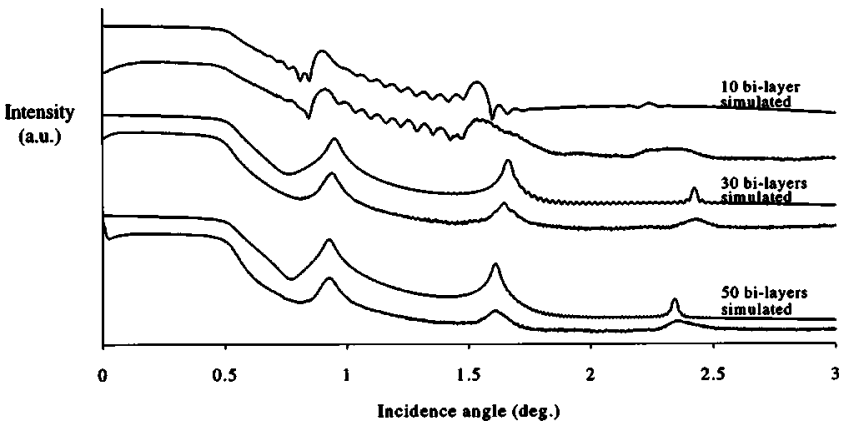

FIG. 2. Experimental (lower curve) and simulated (upper curve) reflectivity spectra of the $\mathrm{Co} / \mathrm{Au}$ multilayers with different number of bilayer repetitions $N$.

ture shown by the TEM images and to the consequent fluctuations in the layers' thickness. ${ }^{8,9}$ Moreover, although the average bilayer thickness deduced from the simulations agrees with the nominal values within the experimental uncertainties $(5 \%)$, to simulate the broadening of the Bragg peaks it was necessary to introduce an interface roughness value comparable with that of the Co layer thickness (around $0.9 \mathrm{~nm}$ ). In addition, the modeled surface roughness (about 2 $\mathrm{nm}$ ) is nearly as high as the thickness of the gold cap layer, probably because the roughness parameters are influenced by the wide waviness of the multilayers evidenced by TEM. The waviness of the film surface also contributes to the disappearance of the Kiessig fringes in the 30 and 50 bilayer spectra.

From the refinement of the XRD patterns by SUPREX ${ }^{10}$ it was possible to deduce the presence of a strong [111] orientation of the Au layers and of appreciable strain in the Co layers, in agreement with previous reports for similar multilayers. ${ }^{3}$ The refinement of the position and shape of the satellite peaks allowed us to deduce the average layer's thickness and its fluctuations. The modeled thicknesses are in agreement with the XRR, TEM, and nominal values within the experimental errors. Higher thickness fluctuations were obtained for the ten bilayers film with respect to the other samples, confirming the improvement of the multilayer's quality with an increasing number of repetitions as inferred from TEM and XRR experiments.

The room temperature hysteresis loops of the multilayers measured in the parallel and perpendicular configurations are shown in Fig. 3. In spite of the waviness of the layered structure resulting from the columnar growth, all the samples display perpendicular anisotropy. An enhancement of the perpendicular anisotropy is observed on increasing the repetition number $N$.

The AGFM results are confirmed by the detailed MFM study of magnetic domains both in the as-prepared state and at remanence, after applying a magnetic field of $10 \mathrm{kOe}$ both perpendicularly and parallel to the sample plane. The micromagnetic structure in the as-prepared state (not shown here) for $N=50$ and $N=30$ is characterized by a much higher contrast than for $N=10$. In fact, the increasing number of layer repetitions improves perpendicular anisotropy. This is also visible in the MFM images taken at polar remanence, reported in the insets of Fig. 3: samples with $N=50$ or $N$ 


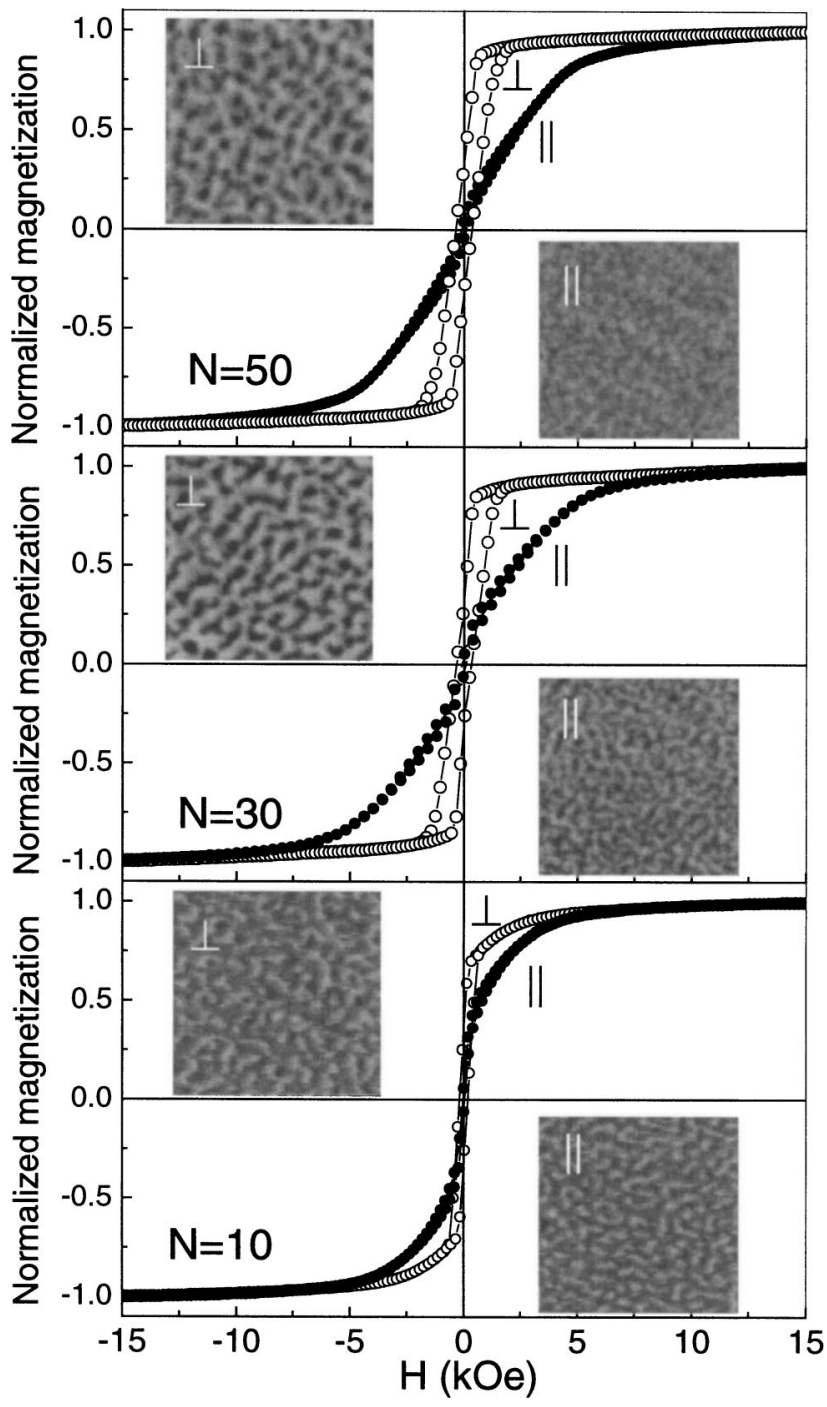

FIG. 3. Parallel $(\|)$ and perpendicular $(\perp)$ normalized hysteresis loops for the $\mathrm{Co} / \mathrm{Au}$ multilayers with different number of repetitions $N$. For each multilayer, MFM images measured at remanence, after the application of a parallel $(\|)$ and a perpendicular $(\perp)$ magnetic field of $10 \mathrm{kOe}$, are shown as insets. Scanning area is $5 \mu \mathrm{m} \times 5 \mu \mathrm{m}$. The light areas indicate domains with magnetization pointing out of the sample plane, dark areas are domains with magnetization pointing into the plane.

$=30$ consist of a larger area of dark part and smaller area of bright part. This asymmetry means the samples remain nonzero magnetization, i.e., have strong remanences. In contrast, for the case of $N=10$ the areas of dark and bright parts are similar and many parts are in the midrange, consistently with the almost zero remanent magnetization. As for longitudinal remanence images, they appear to have a similar portion of dark and bright areas for all the samples.

To achieve a quantitative determination of the effective anisotropy constant for the $\mathrm{Co} / \mathrm{Au}$ multilayers, we measured the dependence of the dipolar spin waves on the external magnetic field strength by Brillouin light scattering. In the magnetic field range between 5 and 12 kOe, shown in Fig. 4, the measured spin wave frequency has an almost linear be-

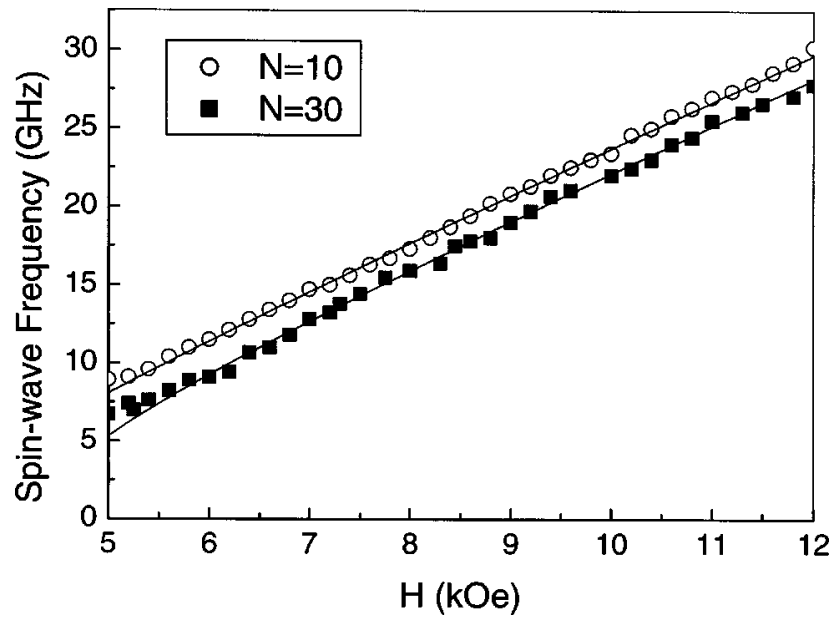

FIG. 4. Spin-wave frequency dependence on the magnetic field intensity for multilayers with different number of repetitions $N=10$ and $N=30$. The data for the $N=50$ multilayer are not shown because they are very close to those of the $N=30$ sample.

havior. These frequencies are appreciably decreased in the specimens with $N=30$ and 50 , with respect to the multilayer with $N=10$, indicating a weaker perpendicular anisotropy in the latter. From a best-fit procedure of the experimental frequencies to those calculated following the procedure described in Ref. 11, the value of the effective anisotropy constant $K^{\text {eff }}$ has been obtained for each multilayer. An appreciable increase of $K^{\text {eff }}$ has been found passing from $N$ $=10\left(K^{\mathrm{eff}}=1.65 \times 10^{6} \mathrm{erg} / \mathrm{cm}^{3}\right) \quad$ to $\quad N=30\left(K^{\mathrm{eff}}=1.96\right.$ $\left.\times 10^{6} \mathrm{erg} / \mathrm{cm}^{3}\right)$, while a very small variation between $N$ $=30$ and $N=50\left(K^{\mathrm{eff}}=1.98 \times 10^{6} \mathrm{erg} / \mathrm{cm}^{3}\right)$ was observed. For all the multilayers $K^{\text {eff }}$ is positive indicating that the magnetization easy axis is perpendicular to the plane and that the tendency toward perpendicular magnetization is enhanced when the repetition number of layers is increased, in agreement with the results obtained by AGFM and MFM.

This work was partially supported by the Nanofilm project of the Istituto Nazionale per la Fisica della Materia (INFM).

${ }^{1}$ D. E. Speliotis, J. Magn. Magn. Mater. 193, 29 (1999).

${ }^{2}$ F. Albertini, G. Carlotti, F. Casoli, G. Gubbiotti, H. Koo, and R. D. Gomez, J. Magn. Magn. Mater. 240, 256 (2002).

${ }^{3}$ G. Gubbiotti, G. Carlotti, F. Albertini, F. Casoli, E. Bontempi, L. E. Depero, H. Koo, and R. D. Gomez, to appear in Thin Solid Films.

${ }^{4}$ S. Hamada, N. Hosoito, T. Ono, and T. Shinjo, J. Magn. Magn. Mater. 198, 496 (1999).

${ }^{5}$ V. Grolien et al., Phys. Rev. Lett. 71, 3023 (1993).

${ }^{6} \mathrm{http}: / /$ ghost.fisica.unipg.it

${ }^{7}$ Version 2.0, Copyright Bruker AXS, 1999.

${ }^{8}$ D. K. G. de Boer, A. J. G. Leenaers, M. W. J. van der Wielen, M. A. Cohen Stuart, G. J. Fleer, R. P. Nieuwhof, A. T. M. Marcelis, and E. J. R. Sudhölter, Physica B 248, 274 (1998).

${ }^{9}$ E. Bontempi, L. E. Depero, L. Sangaletti, F. Giorgis, and C. F. Pirri, J. Mater. Res. 16, 2556 (2001).

${ }^{10}$ E. E. Fullerton, I. K. Schuller, H. Vanderstraeten, and Y. Bruynseraede, Phys. Rev. B 45, 9292 (1992).

${ }^{11}$ L. Albini, G. Carlotti, G. Gubbiotti, L. Pareti, G. Socino, and G. Turilli, J. Magn. Magn. Mater. 198, 363 (1999). 